Journal für Mobilität und Verkehr

ISSN 2628-4154

www.dvwg.de

\title{
Verkehrsverlagerung im Güterverkehr: wichtigster Aspekt im grenzüberschreitenden Verkehr
}

\author{
Dr. Peter Vollmer \\ Postfach, $\mathrm{CH}-3001$ Bern, Schweiz
}

\begin{abstract}
Die Massnahmen für eine Verlagerung des Güterverkehrs sind die grössten und wichtigsten Herausforderungen im grenzüberschreitenden Güterverkehr. Mit der Zustimmung der Bevölkerung zu einer mittels eines Volksbegehrens angeregten, radikalen Verfassungsänderung wurde eine Verlagerungspolitik angestossen, welche seinesgleichen sucht! Die direktdemokratische Mitwirkung der Bevölkerung hat die Behörden gezwungen, die Verlagerungspolitik im Güterverkehr von der Strasse auf die Schiene mit einer Vielzahl von Massnahmen voranzutreiben.
\end{abstract}

Schlagwörter/Keywords: Alpenquerender Güterverkehr, Mitwirkung der Bevölkerung

\section{Einleitung}

Die Schweiz wird in den verkehrswissenschaftlichen Debatten immer wieder als positives Vorbild für eine nachhaltige Verkehrspolitik erwähnt. Dabei stehen verschiedene Massnahmen im Vordergrund, insbesondere das Konzept «Bahn 2000», welches eine flächendeckende Versorgung bis in die ländlichen und alpinen Besiedelungen sicherstellt. Wobei neben einer schweizweiten Vertaktung die fahrplantechnische Verknüpfung aller Verkehrsträger (Eisenbahnen, Busse, Schiffe und Seilbahnen) untereinander ermöglicht, dass notwendige Umsteigemöglichkeiten immer schlank sichergestellt sind.

Ein weiterer, ausserhalb der Schweiz weniger bekannter Meilenstein stellt die Verlagerungspolitik im alpenquerenden Güterverkehr dar. Eine diesbezüglich konsequente und wirkungsvolle Politik wurde durch die Bevölkerung - gegen den Willen der Regierung und des Parlamentes! - mittels eine Volksabstimmung angestossen.

\section{Ausgangslage im alpenquerenden Güterverkehr}

Die topografischen Bedingungen welche bis in anfangs der achtziger Jahre lediglich mittels einer komplizierten Pass- strasse überwunden werden konnten und das erst noch nur in der Sommerzeit zwangen den Güterverkehr auf eine eher komplizierte Verladung auf die Eisenbahn. Die topografischen Voraussetzungen waren in der Vergangenheit sozusagen ein ausreichender Schutz vor einem überquellenden Verkehr. Demgegenüber ermöglichte beispielsweise der Eisenbahn-Gotthard-Scheiteltunnel (erbaut 1880) mit vielen Kunstbauten und sogar sog. Kehrtunnels innerhalb des Berges, einen reibungslosen Verkehr zu allen Jahreszeiten.

Für PKWs ermöglichte nach dem Aufkommen des Automobilverkehs ein Autoverladesystem auf die Eisenbahn, eine Automobilanbindung der Südschweiz an die Alpennordseite, was vor allem staatspolitisch von grösster Bedeutung war. Ein entsprechendes Autoverladesystem existiert und funktioniert bis heute auf der Lötschbergachse für die Verbindung zwischen dem Kanton Bern und dem deutschsprachigen Oberwallis, wo es überhaupt keine dazugehörende Passstrasse gibt, was ohne Autoverlad einen beträchtlichen Umweg über den Kanton Waadt und das Unterwallis erfordert.

Das damit entstandene Monopol-Geschäft der SBB (Schweizerische Bundesbahnen) war sozusagen eine sichere «cashcow», ohne unmittelbare Konkurrenz sowohl bezüglich des Verkehrsmittels noch bezüglich der Route. 
Erst mit dem Ausbau eines Gotthard-Strassen-ScheitelTunnels wurde es für den Schwerverkehr überhaupt erst möglich, diese für viele europäische Güterverkehrsströme attraktive Route zu nutzen. Das Wachstum im alpenquerenden Güterverkehr setzte erst damit überhaupt ein. Der Modal-Split verschob sich kontinuierlich zugunsten der Strasse. Der polemische Satz: «Wer Strassen baut, wird Verkehr ern- ten» lässt sich an diesem Beispiel bestens dokumentieren. Weitergezogene Restriktionen wie das Nacht- und Sonntagsfahrverbot, die Tonnagenbeschränkung pro LKW sowie die geringeren Fahrzeugbreiten konnten keinen grösseren Dämpfungseffekt erzielen und konnten nur bezüglich des Nacht- und Sonntagsfahrverbot überleben.

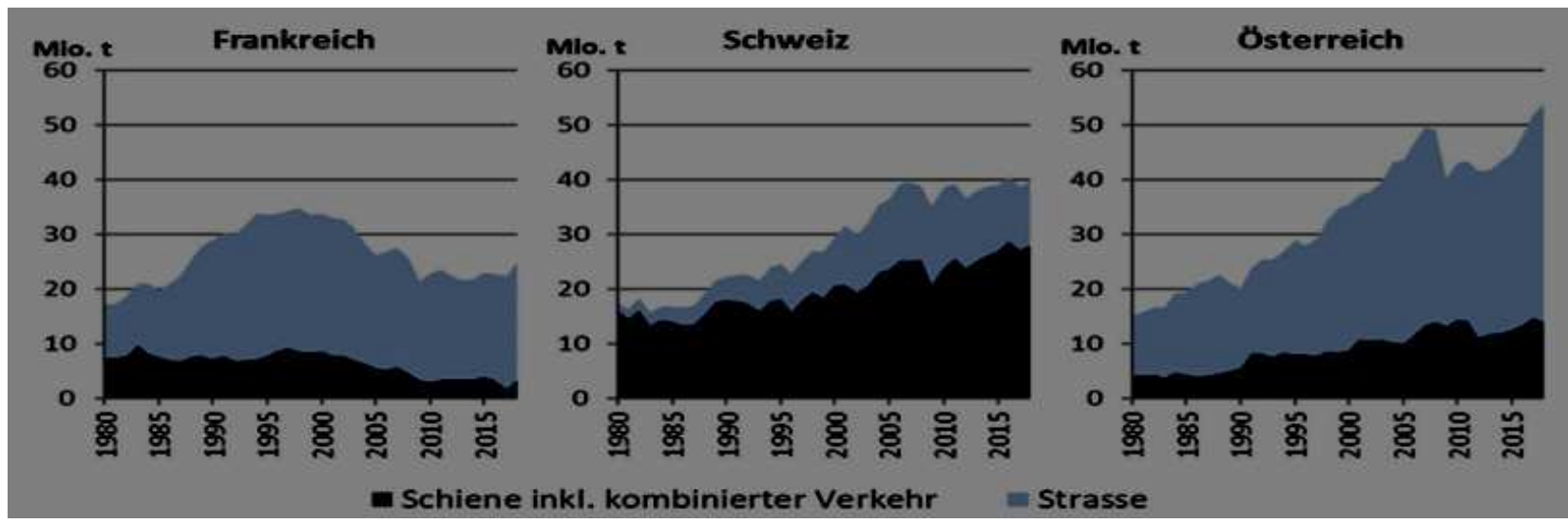

Die sich im schweizerischen Transitkorridor laufend verbesserten Anteile der Schiene basieren ganz wesentlich auf gesellschaftspolitischen Faktoren.

\section{These: Die direktdemokratischen Elemente tragen we- sentlich zum Erfolg der Verlagerung des alpenquerenden Güterverkehrs in der Schweiz bei.}

Vor dem Hintergrund der in Deutschland gemachten Erfahrungen im Umgang mit Grossprojekten (Berliner Flughafen, Stuttgart 21 u.a.m.) werden Bürgerbewegungen und die sog. Zivilgesellschaft oft lediglich als lästige Bremse und als Verzögerungselement wahrgenommen, was sie in einzelnen Fällen zweifellos auch sein können.

Es ist davon auszugehen, dass die schweizerische Bundesregierung und die Parlamentsmehrheit dem europäischen Druck zur Erhöhung der 28-Tonnen-Limite längst nachgegeben hätte, wäre diese Tonnenlimite nicht in einem Bundesgesetz verankert, womit jegliche Änderung mittels einer nicht prohibitiv hohen Unterschriftenzahl zu einer verbindlichen Volksabstimmung gezwungen werden kann. Über das Resultat hätte man auch im Voraus nicht lange rätseln müssen. LKWs sind auch bei vielen PKW-FahrerInnen nicht sonderlich beliebt. Diese sog. Vorwirkung bei den Volksrechten führt dazu, dass es darüber schlussendlich gar nicht zu einer Volksabstimmung kommt.

Österreichische Regionalpolitiker haben selber auch längst Alarm geschlagen, da ein Teil des Verkehrs über die Brennerroute ausgewichen ist und Österreich damit Opfer eines ökologisch fragwürdigen - Umwegverkehrs wurde.

\section{Durchbruch dank der sog. Alpeninitiative!}

Den entscheidenden Durchbruch ermöglichte aber erst die sog. Alpeninitiative. Ein in der Bundesverfassung vorgezeichnetes, verbindliches Volksrecht der direkten Demokratie, welches sich in der Umsetzung jedoch als Knacknuss sondergleichen entpuppte.

Zentrale Inhalte des Volksbegehrens für eine Ergänzung der Bundesverfassung waren:

1. Der Bund schützt das Alpengebiet vor negativen Auswirkungen des Transitverkehrs.

2. Der alpenquerende Gütertransitverkehr von Grenze zu Grenze erfolgt auf der Schiene.

3. Die Transitstrassen-Kapazität im Alpengebiet darf nicht erhöht werden.

4. Die Verlagerung des Gütertransitverkehrs muss zehn Jahre nach Annahme abgeschlossen sein.

1994 wurde der neue Verfassungstext mit einer Bevölkerungsmehrheit von 52\% und der notwendigen Zustimmung der Kantone von 19 der 26 Kantone gutgeheissen. Ein Paukenschlag gegen die Verkehrspolitik von Regierung und Parlament. Diese Mehrheit kam nicht zuletzt auch durch eine «Unheilige Allianz» von UmweltschützerInnen, VerkehrspolitikerInnen, BerggebietseinwohnerInnen und «Nationalistlnnen» zustande.

Wenn - wie dies im konkreten Beispiel der Fall war - die Umsetzungsvorgaben nicht im Verfassungstext direkt vorgeschrieben wurden, hat das Parlament einen grossen Spiel- 
raum bezüglich der Massnahmen zur Erreichung der Zielsetzung. Kommt hinzu, dass es in der Schweiz keine abstrakte Normenkontrolle durch eine Verfassungsgerichtsbarkeit gibt. Zielsetzungen auf Verfassungsstufe können somit auch unerfüllt bleiben.

Eine den Volksentscheid respektierende Umsetzung war wohl demokratiepolitisch eine besonders grosse Knacknuss. Die einfachste und wohl billigste Lösung auf dem Papier wäre ein simples Aufstellen von Verbotsschildern für LKWs im Transit durch die Alpen. Also eine Umsetzung einfach mit Polizeimassnahmen.

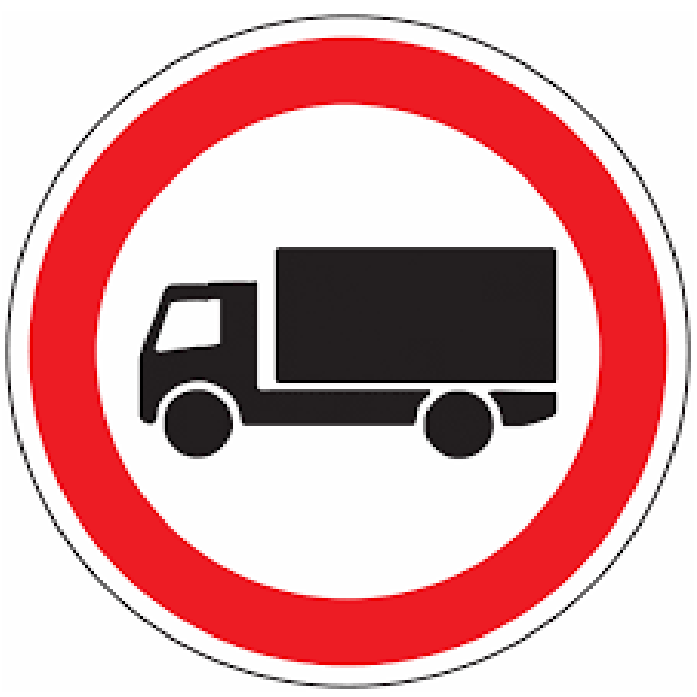

Damit wären jedoch alle ausländischen LKW massivst diskriminiert worden und hätten völkerrechtliche und vertragliche Vereinbarungen für einen freien Zugang missachtet. Mit Retorsionsmassnahmen von Seiten der EU und der Nachbarländer hätte man mit grösster Bestimmtheit rechnen müssen. Die schweizerische Europapolitik befand sich ohnehin bereits in einer heiklen Lage, nachdem das Vertragswerk zur Schaffung eines gemeinsamen Wirtschaftsraums zwischen den EFTA-Staaten und der EU in einer Volksabstimmung im Jahre 1992 abgelehnt wurde.

\section{Gefragt: Politische Kreativität!}

Die Aufgabenstellung war klar, obwohl die Verfassungsvorgabe «Verlagerung von Grenze zu Grenze» im Widerspruch zum Diskriminierungsverbot in der gesamteuropäischen Verkehrspolitik stand. Vor diesem Hintergrund wurde eine Verlagerungspolitik entwickelt, welche den Kern des neuen Verfassungstextes respektierte.

Ein Blick in das Mengengerüst des gesamten alpenquerenden Verkehrs ermöglichte eine neue Betrachtungsweise.

Im Jahre 1994 + 1995, also unmittelbar nach der Abstimmung über die Alpeninitiative präsentierte sich grob gerechnet folgende Ausgangslage im alpenquerenden Verkehr:
- $\quad$ LKW im Transit (Grenze zu Grenze) 650000 Fahrzeuge / pro Jahr

- $\quad$ LKW im Import oder Export 325000 Fahrzeuge / pro Jahr

- $\quad$ LKW im schweizerischen Binnenverkehr 325000 Fahrzeuge / pro Jahr

- Total

1300000 Fahrzeuge / pro Jahr

Vor dieser Ausgangslage wurde folgende Umsetzungsstrategie entwickelt, welche dem Inhaltskern der neuen Verfassungsbestimmung Rechnung tragen soll:

- $\quad$ Der Alpenschutz und die Reduktion des alpenquerenden LKW-Verkehrs sind an sich nicht abhängig vom LKWNummernschuld oder von der Herkunft der Fahrzeuge.

- Mit der Verfassungsbestimmung wird jedoch eine quantifizierbare Anzahl von Fahrzeugen anvisiert, nämlich die 650000 Fahrzeuge welche von Grenze zu Grenze verkehren.

- Aus dem Total der 1300000 Fahrzeuge werden die 650 000 Transitfahrzeuge herausgerechnet, so dass zukünftig nur noch 650000 Fahrzeuge alpenquerend verkehren dürfen. Unabhängig von der Herkunft, resp. der Zuordnung in Transit-, Import/Export- oder Binnenverkehr.

Damit kann dem «Volkswillen», 650000 Fahrten auszusperren, ebenso Rechnung getragen werden.

Gefragt waren damit Reduktionsmassnahmen für alle Kategorien im alpenquerenden Verkehr, welche sich damit nicht mehr diskriminierend gegenüber dem Transitverkehr auswirken.

\section{Breiter Katalog von Reduktionsmassnahmen}

Die Erreichung des vorgegebenen Reduktionsziels soll durch eine Vielzahl von Massnahmen möglich werden, welche grösstenteils in einem besonderen Verlagerungsgesetz festgeschrieben sind. Wichtig unter demokratiepolitischen Aspekten ist das Faktum, dass die seinerzeitigen Initianten der Volksinitiative diesem Umsetzungsweg in der politischen Diskussion ausdrücklich zugestimmt haben.

- Kernstück ist dabei die Attraktivierung des Güter-Schienenverkehrs, welche teilweise schon vor der Abstimmung über die Alpeninitiative eingeleitet wurden: Der Bau der drei Basistunnels (Lötschberg, Gotthard und Ceneri), mit der die Eisenbahn im Alpengebiet zu einer Flachbahn wurde.

- $\quad$ Gleichzeitig wird mit der Modernisierung und Digitalisierung der Bahninfrastruktur die Kapazität und Zuverlässigkeit des Güterverkehrs gefördert.

- Mit der sog. Bahnreform und dem damit geförderten 
Wettbewerb unter den Eisenbahnunternehmungen konnte die Effizienz des Güter-Schienenverkehrs klar gesteigert werden und damit ebenfalls ein wichtiger Beitrag zur Verlagerung geleistet werden.

- Mit den Ausbauten der Zulaufstrecken auf eine 4-Meter-Eckhöhe wird sichergestellt, dass auch sog. Huckepack-Lösungen mit einer rollenden Landstrasse möglich wurden.

- Zur Förderung des kombinierten Verkehrs mit Containern werden Umschlagplätze in Deutschland und Italien mit gefördert.

- $\quad$ Eine grosse Bedeutung für die Verlagerungspolitik ist dabei das sog. Landverkehrsabkommen mit der Europäischen Union, welche der Schweiz weiterhin ermöglicht das Nacht- und Sonntagsfahrverbot im alpenquerenden Güterverkehr aufrecht zu erhalten und vor allem eine Leistungsabhängige Schwerverkehrsabgabe einzuführen.

Kernstück des Landverkehrsabkommens ist zweifelsohne die Anerkennung einer «Leistungsabhängigen Schwerverkehrsabgabe» (LSVA), welche unter anderem auch den Produktionsgewinn der vereinbarten Tonnage-Erhöhung auf 40 Tonnen «wegsteuert» und zusätzliche Steuereinnahmen für den Bahnausbau sicherstellt.

- $\quad$ Eine Strassentransitfahrt zwischen Basel und Chiasso betrug bei Beginn im Jahre 2001 CHF 325 und wurde danach sowohl der Teuerung wie auch den unterschiedlichen Abgasnormen angepasst. Wichtig ist dabei zu erwähnen, dass diese LSVA nicht nur für die alpenquerenden Transitfahrten erhoben wird, sondern im gesamten, auch innerschweizerinnen Verkehr zur Anwendung kommt.

Dazu brauchte es entsprechende Volksabstimmungen, welche trotz Widerstand der LKW-Verbände, gutgeheissen wurden. Die Hinweise auf eine allgemeine Verteuerung der transportierten Güter hat sich nicht zuletzt dank der Anwendung von intelligenteren Logistiklösungen und der Vermeidung von Leerfahrten nicht im befürchteten Ausmass erwiesen.

Der Fokus aller Massnahmen zielt darauf hin, die Wettbewerbsfähigkeit der Schiene zu stärken und damit die Verlagerung sowohl preislich wie logistisch sicher zu stellen. Die Wahl des Transportmittels ist für die grossen privaten Güterverkehrs-Unternehmngen keine ideologische Frage, sondern wird in erster Linie durch den Preis bestimmt, wobei ebenso die Zuverlässigkeit, die Angebots- und TransportQualität und die logistischen Bedingungen bestimmend sind. Die Verlagerung kann deshalb in einem offenen System nur dann gelingen, wenn die Bedingungen bei der Eisenbahn entsprechend besser werden.
Die Speisung des neu geschaffenen Bahninfrastrukturfonds $(B F I)$, wird dabei nicht allein durch zwei Drittel der Erträge aus der LSVA, sondern unter anderem auch durch die Übertragung eines Promille der gesamten Mehrwertsteuererträge sicher gestellt. Dadurch wurde ein für die Eisenbahn wichtiger Grundsatz umgesetzt, dass gerechtfertigt durch eine Gesamtbetrachtung in der Verkehrspolitik StrassenSteuererträge direkt zur Schiene umgelagert werden.

\section{Alpentransitbörse als Lösungsweg?}

Vor dem Hintergrund der nur langsam wirkenden Massnahmen der Verlagerung, womit die verfassungsrechtlich festgeschriebenen Vorgaben (Umsetzung der Vorgaben innert 10 Jahren) wurden sowohl von Seiten der Initianten der erfolgreichen Volksinitiative wie auch von Seiten der Bundesregierung die Schaffung einer sog. Alpentransitbörse in die Diskussion gebracht. An dieser Börse würden die verknappten alpenquerenden Fahrten (650 000 Fahrten pro Jahr) frei gehandelt. Diese aus rein marktwirtschaftlicher Sichtweise bestechende Lösung würde jedoch zweifellos zu einer deutlichen Steigerung der einzelnen Durchfahrtspreise führen und wäre damit für die Anpassung des bilateralen Landverkehrsabkommen nicht durchsetzungs- und damit nicht konsensfähig. Dennoch hat die Schweizer Regierung einen parlamentarischen Auftrag, entsprechende Sondierungen bei der Europäischen Union wie auch bei den Nachbarstaaten aufzunehmen.

\section{Fazit}

Das Beispiel der Verlagerungspolitik in der Schweiz offenbart verschiedene Erkenntnisse:

- $\quad$ Eine wirkungsvolle Verlagerungspolitik lässt sich nur durch eine Vielzahl von Massnahmen erreichen.

- Die Verlagerungspolitik ist ein längerfristiger Prozess, der durch die Dynamik in den Entwicklungen und Innovationen der Gütertransporte sowohl auf der Strasse, wie auf der Schiene immer wieder neu definiert und angepasst werden muss.

- Eine wirkungsvolle Verlagerungspolitik muss von der betroffenen Bevölkerung nicht nur verstanden, sondern noch besser direkt initiiert werden, damit diese dann auch in der Umsetzung mitgetragen wird. Das schweizerische Verfassungsrecht sieht dafür mit den direktdemokratischen Möglichkeiten (Verfassungsinitiative und Gesetzesreferendum) entsprechende Instrumente vor.

- Die direktdemokratischen Elemente tragen wesentlich zum (Teil-)Erfolg der Güterverkehrsverlagerung im Alpenraum bei. 


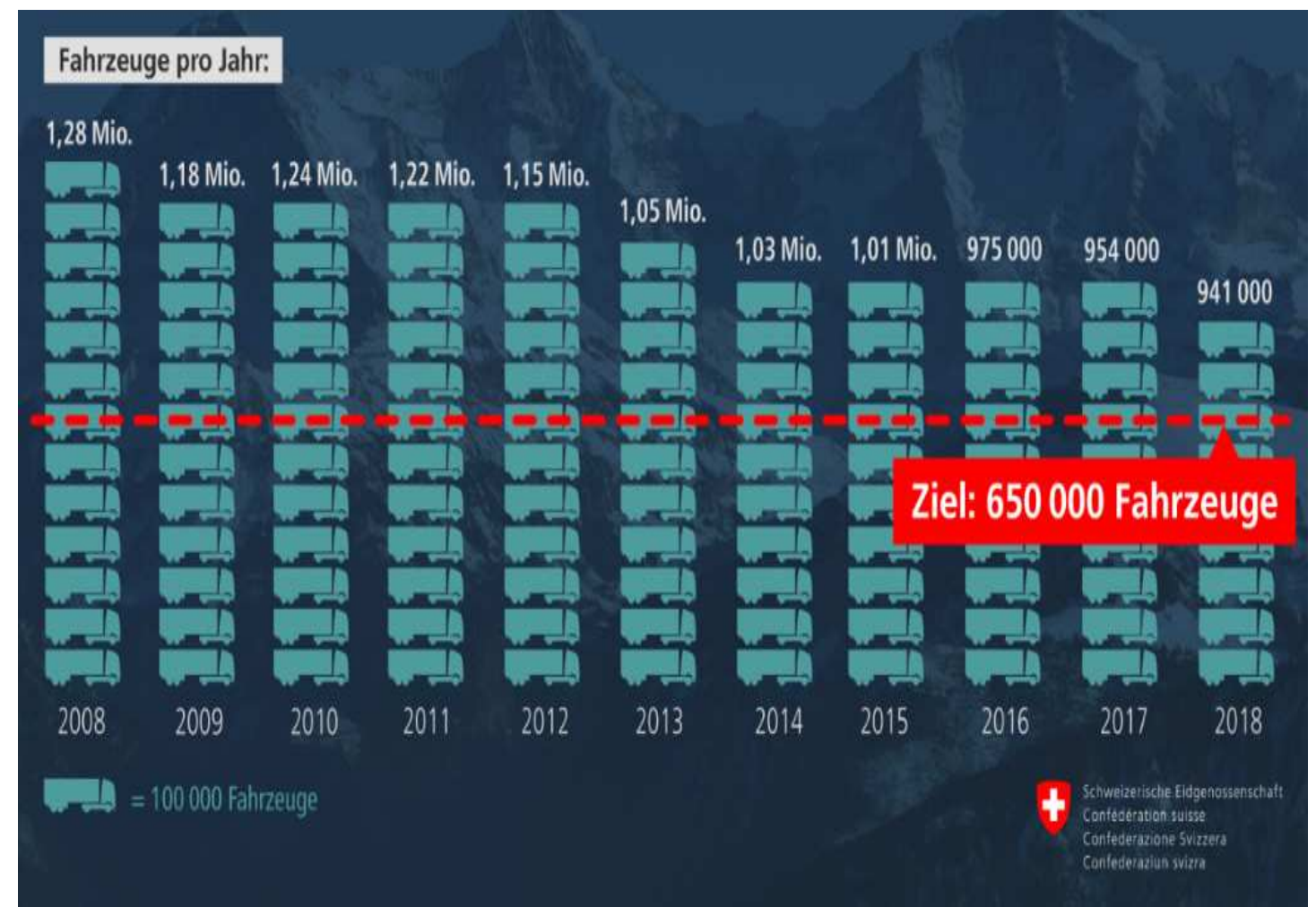

\section{Literatur- und Quellenverzeichnis}

Alpenquerender Güterverkehr durch die Schweiz. Kennzahlen 1. Halbjahr 2021. Schweizerische Eidgenossenschaft. UVEK, BAV Datenkompetenzzentrum Güterverkehr. September 2021.

Verlagerung des Güterverkehrs durch die Alpen. Bundesamt für Verkehr (BAV). Faktenblatt 2021. www.bav. admin.ch.

Bericht über die Verkehrsverlagerung vom November 2021. Verlagerungsbericht Juli 2019 - Juni 2021. Schweizerische Eidgenossenschaft. Bericht des Bundesrates. Bundesamt für Verkehr 2021.

Gesamtschau der Interessen der Schweiz im Strassen-, Schienen- und Luftverkehr in den grenzüberschreitenden Beziehungen mit Deutschland. Bericht des Bundesrates. Schweizerische Eidgenossenschaft, vom 18.6.2021

Bundesgesetz über die Verlagerung des alpenquerenden Güterschwerverkehrs von der Strasse auf die Schiene, Güterverlagerungsgesetz (GVVG) vom 19.12.2008.

Protokoll der Volksabstimmung vom 20.2.1994 über die Eidgenössische Volksinitiative «zum Schutz des Alpengebietes vor dem Transitverkehr». Schweizerische Eidgenossenschaft, Bundeskanzlei 1994.
Tätigkeitsbericht 2020. Kommission für den Eisenbahnverkehr. Schweizerische Eidgenossenschaft (Rail Com). 2021. 